\title{
Microstructural improvements of InP on GaAs (001) grown by molecular beam epitaxy by in situ hydrogenation and postgrowth annealing
}

\author{
F. M. Morales, ${ }^{1, a)}$ R. García, ${ }^{1}$ S. I. Molina, ${ }^{1}$ A. Aouni, ${ }^{2}$ P. A. Postigo, ${ }^{3}$ and C. G. Fonstad ${ }^{4}$ \\ ${ }^{1}$ Dep. Ciencia de los Materiales e IM y QI., F. Ciencias, Universidad de Cádiz, Puerto Real, \\ Cádiz 11510, Spain \\ ${ }^{2}$ Faculté des Sciences et Techniques, Université Abdelmalek Essadi, BP 416 Tanger, Morocco \\ ${ }^{3}$ Instituto de Microelectrónica de Madrid, CNM, CSIC, Tres Cantos, Madrid 28760, Spain \\ ${ }^{4}$ Department of Electrical Engineering and Computer Science, and Microsystems Technology Laboratory, \\ MIT, Cambridge, Massachusetts 02139, USA
}

(Received 6 November 2008; accepted 13 January 2009; published online 30 January 2009)

\begin{abstract}
The characterization of high quality InP on GaAs (001) fabricated by molecular beam epitaxy using a two-step growth method involving hydrogenation during growth is reported. Electron diffraction and high-resolution transmission electron microscopy confirm that $\sim 2 \mu \mathrm{m}$ thick InP epilayers on $\mathrm{GaAs}$ are heteroepitaxial and strain relaxed. Stacking faults and threading dislocations are mostly confined near the InP/GaAs interface and their densities decrease monotonically toward the InP surface. Additionally, rapid-thermal annealing following growth is found to result in a marked reduction in the number of dislocations and the disappearance of planar defects. (C) 2009 American Institute of Physics. [DOI: 10.1063/1.3077610]
\end{abstract}

Indium phosphide (InP) has generated much interest in the past decade due to its superior advantages for optoelectronic integrated circuits, high speed microwave and millimeter wave circuits, solar cells, and wireless applications. ${ }^{1-6}$ The monolithic integration of InP components on $\mathrm{Si}$ or GaAs substrates is limited by the following important issues: it is brittle, its technology is fairly young, and bulk InP wafers are available only in small sizes and cost about four times as much GaAs. ${ }^{6}$ Outstanding progress are being made in the effort to grow thin InP layers on Si (Refs. 7 and 8) but much remains to be done and significant challenges still must be overcome. Consequently, the heteroepitaxy of InP films on GaAs substrates has emerged as an alternate solution to the need for large area InP device heterostructures. To be successful, however, this approach requires defeating the negative effects produced by the high lattice mismatch $(3.8 \%)$ and the very high difference in the thermal expansion coefficients (19.7\%) between InP and GaAs. Indeed, according to the Matthews and Blakeslee model, ${ }^{9}$ the critical layer thickness of InP on GaAs is less than $5 \mathrm{~nm}$.

Several techniques have been used to reduce defects and to improve the crystalline, optical and electronic qualities of InP grown directly on GaAs. In early work, mirrorlike InP surfaces were obtained by metal-organic chemical-vapor deposition (MOCVD). ${ }^{10}$ Growth on sawtooth patterned GaAs substrates demonstrated the reduction in threading dislocations (TDs) because the sawtooth-shaped interfaces increase the probability of TD interactions and self-annihilations. ${ }^{11}$ An improved two-step growth method was also demonstrated in which the V/III ratio was lowered for the growth of an initial low-temperature layer at $400{ }^{\circ} \mathrm{C} .{ }^{12}$ On fused InP/GaAs wafers no threading dislocations developed and the mismatch was accommodated entirely by misfit dislocations (MDs). ${ }^{13-15}$ Derbali et al. ${ }^{16}$ fabricated good quality InP on the less-conventionally used (111) oriented GaAs with a remarkably reduced density of

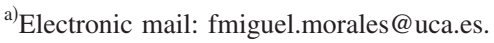

TDs (DTDs). Recently, Liao et al. ${ }^{17}$ and Yarn et al. ${ }^{18}$ have shown high quality InP layers with flat uniform surface morphologies grown directly on GaAs by metal-organic vaporphase epitaxy (MOVPE). The DTD near the top surface of a $1 \mu \mathrm{m}$ thick InP nonannealed layer was $5 \times 10^{7} \mathrm{~cm}^{-2}$. Zhou et al. ${ }^{19}$ have shown that a $120 \mathrm{~nm}$ thick low-temperature InP nucleation layer is effective in significantly reducing the stress in a thick InP layer grown subsequently at the usual higher temperatures.

Using step graded buffers or strained layer superlattices between the epitaxial InP and the GaAs substrate can improve the quality of epilayers dramatically, allowing yielding DTDs as low as $9 \times 10^{5} \mathrm{~cm}^{-2}$ without the use of annealing. Xiong et al. $^{20}$ demonstrated this using an optimized two-step scheme with low-temperature initial conditions in lowpressure MOCVD, and Quitoriano and Fitzgerald ${ }^{21}$ grew good quality InP this way by atmospheric pressure MOCVD.

The technique of direct growth of InP on GaAs by molecular beam epitaxy (MBE) using monoatomic hydrogen $\left(\mathrm{H}^{*}\right)$ has also recently demonstrated excellent results. ${ }^{22}$ For $\mathrm{H}^{*}$-assisted MBE, the critical thickness, as observed by reflection high-energy electron diffraction, increased to at least $200 \mathrm{~nm}$, a value which is $\sim 40$ times larger than expected. Additionally, these heteroepitaxial InP films showed good optical properties, with a band-to-band intensity at room temperature nearly one fourth the intensity for homoepitaxial InP grown under the same conditions. For some specimens a rapid-thermal annealing (RTA) cycle after epitaxy was required to achieve this result but with certain doses of hydrogen and no RTA was needed to obtain the same photoluminescence (PL) response at room temperature. To explore these results further, a microstructural assessment of InP layers grown on GaAs by $\mathrm{H}^{*}$-assisted MBE using different $\mathrm{H}^{*}$ doses was undertaken. In particular, the contributions of the $\mathrm{H}^{*}$ and RTA processes to the reduction in extended structural defects in the InP layers were examined.

InP epilayers $2 \mu \mathrm{m}$ thick were fabricated by lowtemperature two-step solid-source MBE on (001) GaAs wa- 


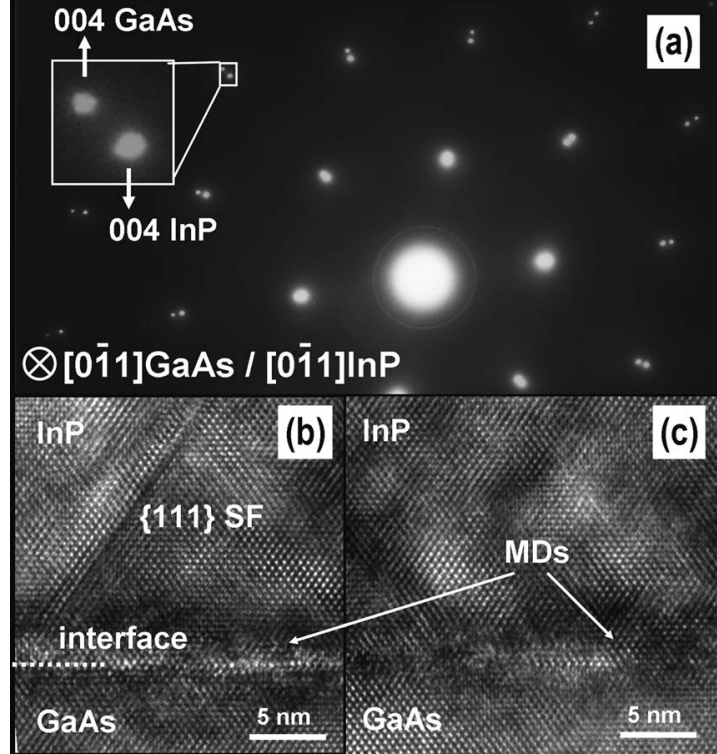

FIG. 1. (a) XTEM-SAED pattern showing both GaAs and InP aligned associated reflections. HRTEM images of the GaAs/InP interface: (b) The InP of sample A1 shows a SF, (c) and that of sample C2 is clean of defects.

fers. The first step of deposition at $200{ }^{\circ} \mathrm{C}$ is, in some cases, assisted by $\mathrm{H}^{*}$ (cracked from $\mathrm{H}_{2}$ with a filament at $2000{ }^{\circ} \mathrm{C}$ ) at beam equivalent pressures of $1 \times 10^{-5}$ mbar (medium $\mathrm{H}^{*}$ ) or $5 \times 10^{-5}$ mbar $\left(\right.$ high $\mathrm{H}^{*}$ ). The use of $\mathrm{H}^{*}$ was stopped at an InP thickness of $\sim 250 \mathrm{~nm}$ and the subsequent InP growth happened at $450{ }^{\circ} \mathrm{C}$ and without $\mathrm{H}^{*}$. Six samples were analyzed; three of them without $\mathrm{H}^{*}$ (A1) or using the medium (B1) or the high dose of $\mathrm{H}^{*}(\mathrm{C} 1)$; the other 3, named A2, B2, and $\mathrm{C} 2$, were grown similarly but were also subjected to postannealing. This RTA involved rapidly heating to $780{ }^{\circ} \mathrm{C}$ for $10 \mathrm{~s}$, followed by a fast cooldown. Preliminary highresolution $\mathrm{x}$-ray diffraction (XRD) analyses indicated a high relaxation rate $(99.1 \%)$ for samples $\mathrm{B} 1$ and $\mathrm{C} 1$, and even higher relaxation (between $99.6 \%$ and $99.8 \%$ ) for the other samples, indicating they are almost fully strain relaxed. The growth and annealing conditions and the XRD results are detailed elsewhere. ${ }^{22}$

The heterostructures were structurally characterized by transmission electron microscopy (TEM) techniques: selected area electron diffraction (SAED) and high-resolution TEM (HRTEM), carried out in JEOL thermoionic and fieldemission microscopes (a JEM-1200EX, operated at $120 \mathrm{keV}$, and a JEM-2010F, operated at $200 \mathrm{keV}$, respectively). The cross-sectional TEM (XTEM) and plan-view TEM (PVTEM) sample preparations consisted of classical routines of mechanical thinning, plus ion milling and subsequent plasma cleaning.

The studies of SAED patterns confirm that the InP layers are single-crystalline and well heteroepitaxially placed as indicated by the perfect alignments between GaAs and InP diffraction lattice spots. Figure 1(a) shows an XTEM-SAED pattern for sample B2. The GaAs and the InP related sharp reflections are aligned, and this configuration was seen for every sample studied. In addition, no arcing of these spots, extra spots, or different faint contrasts, the presence of which could indicate either mosaicity in the layer or a high quantity of extended planar defects [stacking faults (SFs) or twins], were found. As demonstrated by XRD, we conclude from the analyses of these diffractograms and the study of the GaAs/

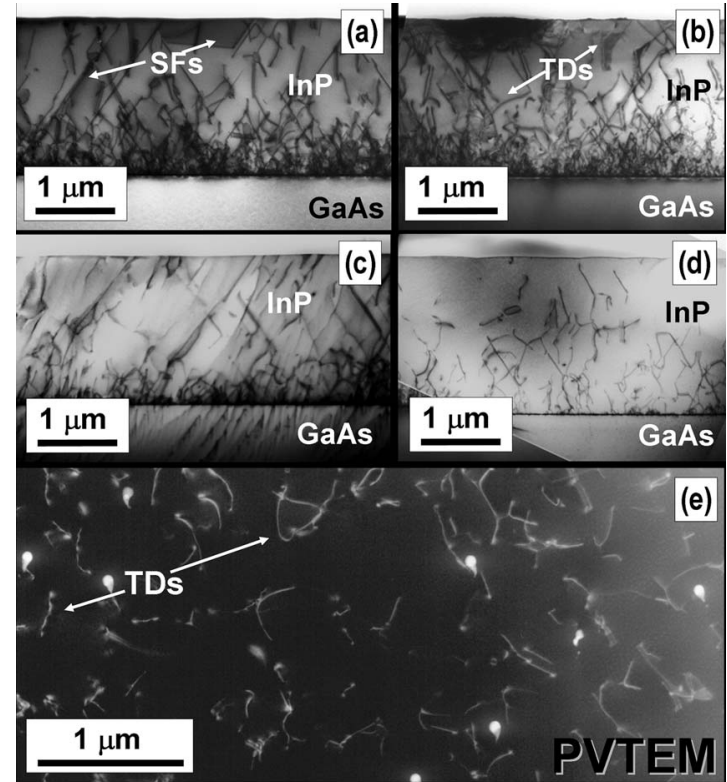

FIG. 2. Bright-field two-beam diffraction-contrast (2B-DC) XTEM images using the reflection $g=004$ (conditions to make TDs visible) of the InP/ GaAs heterostructure of samples A1 (a), C1 (b), B2 (c), and C2 (d), respectively. (e) Dark-field 2B-DC $g=044$ PVTEM micrograph of the InP epilayer of $\mathrm{C} 2$.

InP interfaces in HRTEM micrographs that the InP layers are almost completely strain relaxed. It could also be seen in these images that MDs formed just at the interface after the critical thickness was reached during the growth to relieve the mismatch strain. Moreover, two other main kinds of structural defects, both typical of cubic crystals, were observed: SFs and tangled TDs. Their numbers increased, the further the growth conditions were far from optimum. Figures 1(b) and 1(c) show two HRTEM micrographs of the sharp (001)GaAs/(001)InP interfaces of specimens A1 and $\mathrm{C} 2$, respectively. At this magnification, MDs should be visible at the interface, and while the less-than-optimized InP layer (A1) shows a $\{111\}$ SF, the improved $\operatorname{InP}(\mathrm{C} 2)$ has a structure clean of defects.

Both TDs and SFs imperfections are mostly confined to a region less than $500 \mathrm{~nm}$ above the $\mathrm{InP} / \mathrm{GaAs}$ interface (due to interfacial strain) and their densities are found to decrease monotonically as they approach the surface of the InP films (due to continuous autoannihilation and decreasing stress). The TDs appear irregularly spread across the films and are found to have $\vec{g}$ vectors parallel to $\langle 022\rangle$ or $\langle 004\rangle$ directions, thus indicating a predominant glide on the $\{111\}$ slip planes. ${ }^{18}$ However, planar defects did not appear in the samples with higher $\mathrm{H}^{*}$ doses and the interface region looks cleaner of imperfections. As a rule, it is found that there is a reduction in the DTD of one or two orders of magnitude, respectively, when high $\mathrm{H}^{*}$ doses or RTA are used alone or in combination [see Figs. 2(a)-2(d)]. For the improved sample C2, the DTD was measured to be on the range of $\sim 10^{8} \mathrm{~cm}^{-2}$ [Fig. 2(e)], which is a good result compared to state of the art InP epilayers. ${ }^{18}$ Comparable quantities of such defects have been demonstrated to improve electrical and optical properties by acting as a gettering network located beneath the device zone on $\mathrm{InP} / \mathrm{GaAs}$ based high-electron mobility transistors. ${ }^{23}$ 
At this point one should ask why hydrogenation and annealing bring such beneficial effects. The role of $\mathrm{H}^{*}$ treatment for decreasing the DTD has already been reported for other semiconductors, as for example, in GaAs films on $\mathrm{Si}$ grown by $\mathrm{MBE}^{24}$ In hydrogenated $\mathrm{SiGe} / \mathrm{Si}$ heterostructures grown by MBE the $\mathrm{H}$-induced defects promoted strain relaxation via preferred nucleation of dislocation loops which extend to the interface to form misfit segments. ${ }^{25}$ In $\mathrm{SiGe} / \mathrm{Ge}$ grown by MOCVD, the effect of monoatomic hydrogen was remarkable in the early stages of strain relaxation and during subsequent thermal treatment. ${ }^{26}$ On pulsed laser depositions using a nitrogen-hydrogen gas, Ito $e t a l .^{27}$ found that the PL emission of the grown $\mathrm{GaN}$ was increased, an effect that was attributed most likely to the reduction in DTD. In the system $\mathrm{InP} / \mathrm{GaAs}$ under study, a $\mathrm{H}$ plasma was needed to passivate both $\mathrm{Zn}$ acceptors and TDs and thus to improve the quality and performance of heteroepitaxial InP-based space solar cells. $^{28,29}$ The hydrogenation of semiconductors modifies both chemical reactivity and electrical conductivity, making them less reactive (passivated), and may remove surface states by terminating surface dangling bonds. ${ }^{30}$ These might be some of the reasons for the improved epitaxy (DTD: C1-B2 $\sim 10^{9} \mathrm{~cm}^{-2} ; \quad \mathrm{C} 2 \sim 10^{8} \mathrm{~cm}^{-2}$ ) and the highly improved emission ${ }^{22}$ in the present $\mathrm{H}^{*}$-assisted experiments ( $\mathrm{PL}$ at room temperature similar to $\mathrm{C} 1$ and $\mathrm{C} 2$ ).

On the other hand, thermal cyclic annealing was reported to be effective in reducing the InP DTD by half from $6 \times 10^{7}$ to $3 \times 10^{7} \mathrm{~cm}^{-2}$ at a thickness of $5 \mu \mathrm{m}$ and was essential to confine point defects near the InP/GaAs interface. ${ }^{31}$ Annealing reduced the DTD near the surface of a $3 \mu \mathrm{m}$ layer one order of magnitude, while it was higher than $10^{8} \mathrm{~cm}^{-2}$ for the as-grown layers. ${ }^{12}$ Note that the best present DTD results $\left(\sim 10^{8} \mathrm{~cm}^{-2}\right.$ TDs for a $2 \mu \mathrm{m} \mathrm{InP} \mathrm{layer}$ after RTA) are comparable to the values typical of metamorphically grown InP layers $6 \mu \mathrm{m}$ or thicker ${ }^{18}$ and that just $2 \mu \mathrm{m}$ has been established as the film thickness above, which the TDs amounts start to dramatically decrease with increasing thickness. ${ }^{32}$ However, it is unclear whether a relatively high DTD $\left(10^{6}-10^{8} \mathrm{~cm}^{-2}\right)$ enhances the diffusion of point defects and impurities toward the TD network in metamorphic InP. ${ }^{23}$ Note again that the PL signal is similar in samples $\mathrm{C} 1$ and $\mathrm{C} 2$, although the defect structural differences between these samples are obvious in Fig. 2, indicating that for the same DTD, an InP layer with $\mathrm{H}^{*}$ would have better PL than one without $\mathrm{H}^{*}$.

In conclusion, improvements in the microstructure of InP epilayers on GaAs from the use of $\mathrm{H}^{*}$ during MBE and successive RTA have been demonstrated herein through TEM-based techniques. Using hydrogenation during the early stages of the growth, two orders of magnitude reduction in DTD is achieved with the use of RTA. Planar defects did not appear in the samples with higher $\mathrm{H}$ doses and the interface region appears clear of imperfections. Further work using some of the improved epitaxial growth methods introduced here, in combination with hydrogenation and postannealing, should lead to even more improved InP epilayers in the near future.
This work was supported by the Spanish MEC (Grant Nos. TEC2005-05781-C03-02 and MAT200760643), and MCI (Grant Nos. TEC2008-06756-C03-02), Junta de Andalucía (PAI group TEP-120; Project No. PAI05TEP-00383), and Project Nos. NAN2004-09109-C04-01, TEC-2005-05781-C03-01， CONSOLIDER-Ingenio 2010 CSD2006-00019 QOIT and CSD2006-004 GENESIS-FV. TEM measurements were carried out at DME, SCCYTUniversidad de Cádiz.

${ }^{1}$ U. K. Mishra and J. B. Shealy, Sixth International Conference on InP and Related Materials, 1994 (unpublished), p. 14

${ }^{2}$ N. M. Margalit, J. Piprek, S. Zhang, D. I. Babic, K. Streubel, P. P. Mirin, J. R. Wesselmann, J. E. Bowers, and E. L. Hu, IEEE J. Sel. Top. Quantum Electron. 3, 359 (1997).

${ }^{3}$ L. Goldstein, C. Fortin, C. Starck, A. Plais, J. Jacquet, T. Boucart, A. Roche, and C. Poussou, Electron. Lett. 34, 268 (1998).

${ }^{4}$ R. Szweda, III-Vs Review 13, 55 (2000).

${ }^{5}$ III-Vs Review 18, 27 (2005).

${ }^{6} \mathrm{M}$. Cooke, III-Vs Review 19, 23 (2006).

${ }^{7}$ O. Parillaud, E. Gil-Lafon, B. Gerard, P. Etienne, and D. Pribat, Appl. Phys. Lett. 68, 2654 (1996).

${ }^{8}$ F. Olsson, M. Xie, S. Lourdudoss, I. Prieto, and P. A. Postigo, J. Appl. Phys. 104, 093112 (2008).

${ }^{9}$ J. W. Matthews and A. E. Blakeslee, J. Cryst. Growth 27, 118 (1974).

${ }^{10}$ H. Horikawa, Y. Ogawa, Y. Kawai, and M. Sakuta, Appl. Phys. Lett. 53 397 (1988).

${ }^{11}$ Y. Okuno, T. Kawano, M. Koguchi, K. Nakamura, and H. Kakibayashi, J. Cryst. Growth 137, 313 (1994).

${ }^{12}$ Y. Takano, T. Sasaki, Y. Nagaki, K. Kuwahara, S. Fuke, and T. Imai, J. Cryst. Growth 169, 621 (1996).

${ }^{13}$ H. Wada, Y. Ogawa, and T. Kamijoh, Appl. Phys. Lett. 62, 738 (1993).

${ }^{14}$ Y. Okuno, K. Uomi, M. Aoki, T. Taniwatari, M. Susuki, and M. Kondow, Appl. Phys. Lett. 66, 451 (1995).

${ }^{15}$ L. Sagalowicz, A. Rudra, A. Syrbu, and J. Behrend, Philos. Mag. Lett. 76 445 (1997).

${ }^{16}$ M. B. Derbali, J. Meddeb, H. Maaref, D. Buttard, P. Abraham, and Y Monteil, J. Appl. Phys. 84, 503 (1998).

${ }^{17}$ C. I. Liao, K. F. Yarn, C. L. Lin, Y. L. Lin, and Y. H. Wang, Jpn. J. Appl Phys., Part 1 42, 4913 (2003).

${ }^{18}$ K. F. Yarn, C. I. Liao, and C. L. Lin, Crystallogr. Rev. 12, 47 (2006).

${ }^{19}$ W. Zhou, C. W. Tang, J. Zhou, K. M. Lau, Y. Zeng, H. G. Liu, N. G. Tao, and C. R. Bolognesi, IEEE Electron Device Lett. 28, 539 (2007).

${ }^{20}$ D. Xiong, X. Ren, Q. Wang, J. Zhou, W. Shu, J. Lu, S. Cai, H. Huang, and Y. Huang, Chin. Opt. Lett. 5, 422 (2007).

${ }^{21}$ N. J. Quitoriano and E. A. Fitzgerald, J. Appl. Phys. 102, 033511 (2007)

${ }^{22}$ P. A. Postigo, F. Suarez, A. Sanz-Hervás, J. Sangrador, and C. G. Fonstad, J. Appl. Phys. 103, 013508 (2008).

${ }^{23}$ Y. Liu and H. Wang, J. Appl. Phys. 100, 034505 (2006).

${ }^{24}$ Y. Okada, H. Shimomura, T. Sugaya, and M. Kawabe, Jpn. J. Appl. Phys., Part 1 30, 3774 (1991).

${ }^{25}$ S. Mantl, B. Holländer, R. Liedtke, S. Mesters, H. J. Herzog, H. Kibbel, and T. Hackbarth Nucl. Instrum. Methods Phys. Res. B 147, 29 (1999).

${ }^{26}$ Y. Yamashita, R. Nakagawa, Y. Sakamoto, T. Ishiyama, and Y. Kamiura, Physica B 376, 204 (2006)

${ }^{27}$ S. Ito, H. Fujioka, J. Ohta, A. Kobayashi, T. Honke, H. Mikic, and M. Oshima, Thin Solid Films 457, 118 (2004).

${ }^{28}$ B. Chatterjee, S. A. Ringel, R. M. Sieg, R. Hoffman, and I. Weinberg, Appl. Phys. Lett. 65, 58 (1994).

${ }^{29}$ B. Chatterjee and S. A. Ringel, Appl. Phys. Lett. 69, 839 (1996); S. A. Ringel and B. Chatterjee, J. Appl. Phys. 83, 5904 (1998).

${ }^{30}$ A. J. Mayne, D. Riedel, G. Comtet, and G. Dujardin, Prog. Surf. Sci. 81, 1 (2006)

${ }^{31}$ N. Hayafuji, T. Kimura, N. Yoshida, N. Kaneno, M. Tsugami, K. Mizuguchi, T. Murotani, and S. Ibuki, Jpn. J. Appl. Phys., Part 2 28, L1721 (1989).

${ }^{32}$ D. J. Olego, Y. Okuno, and T. Kawano, J. Appl. Phys. 71, 4492 (1992). 